\title{
A THEORETICAL INVESTIGATION OF AN AEROFOIL EQUIPPED WITH A SPLIT FLAP
}

D. G. HURLEY

(received 29 September 1960, revised 17 December 1960)

\section{NOTATION}

$b \quad$ Parameter, see eqn (2)

$C_{f} \quad$ Length of flap as fraction of chord of wing, see eqn (24)

$C_{D}, C_{L}$ Drag and lift coefficients respectively, see eqns. (24) and (25)

$\mathscr{C}$ Integration contour, see eqn (14)

$d \quad$ Parameter, see eqn (7)

$f, g, h$ Parameters, see eqn (2)

$K$ Constant, see eqn (2)

$L \quad$ Constant, see eqn (3)

$q \quad$ Fluid speed, see eqn (1)

$q_{G} \quad$ Value of $q$ at point $G$.

$Q \quad=\log \left(-U \frac{d z}{d w}\right)$, see eqn (1)

$t \quad$ Auxiliary plane, see Fig. 5.

$u, v \quad$ Velocity components, see page 1 .

$U \quad$ Value of $u$ at infinity

$w \quad$ Complex velocity potential

$x, y$ Co-ordinates in physical plane

$X, Y$ Components of force acting on wing-flap combination

$z \quad=x+i y$

$\alpha \quad$ Incidence, see eqn (4)

$\beta \quad$ Flap deflection, see eqn (4)

$\gamma \quad$ Parameter, see eqn (8)

$\zeta \quad$ Auxiliary variable, see eqn (2)

$\theta \quad$ Direction of fluid velocity

$\rho \quad$ Fluid density

$\phi \quad$ Velocity potential

$\psi \quad$ Stream function 


\section{Introduction}

For a theory of the flow about an aerofoil equipped with a split flap to be satisfactory, account must be taken of the wake which is formed downstream of the combination. In this paper the wing and the flap are each represented by a flat plate and it is assumed that the pressure in the wake is constant and equal to the free stream value, i.e. it is assumed that the wake may be represented by the region between two free stream-line which extend downstream from the trailing edges of the wing and the flap ${ }^{1}$ ) (see Fig. 1).

In this model the width of the wake at the trailing edge of the wing is accurately represented but the assumption that the wake pressure is constant and equal to the free stream value is an idealization. In practice the pressure at the trailing edge of the wing will be less than the free stream value and will only increase to this value well downstream of the wing. Small transverse variations in pressure will also occur. It is to be expected that these effects will lead to the actual drag being greater than the theoretical value. However, their influence on the lift should be less so that the theoretical predictions of this quantity may be reasonably accurate.

\section{Analysis}

The flow in the physical or $z=x+i y$ plane (see Fig. 1) is determined by employing the classical methods of free stream-line theory. The velocity components $u$ and $v$ are given in terms of the complex velocity potential, $w$, by $d w / d z=-u+i v$ and $d w / d z$ is determined as a function of an auxiliary variable $\zeta$ by mapping the $Q$-plane onto the upper half of the $\zeta$-plane where

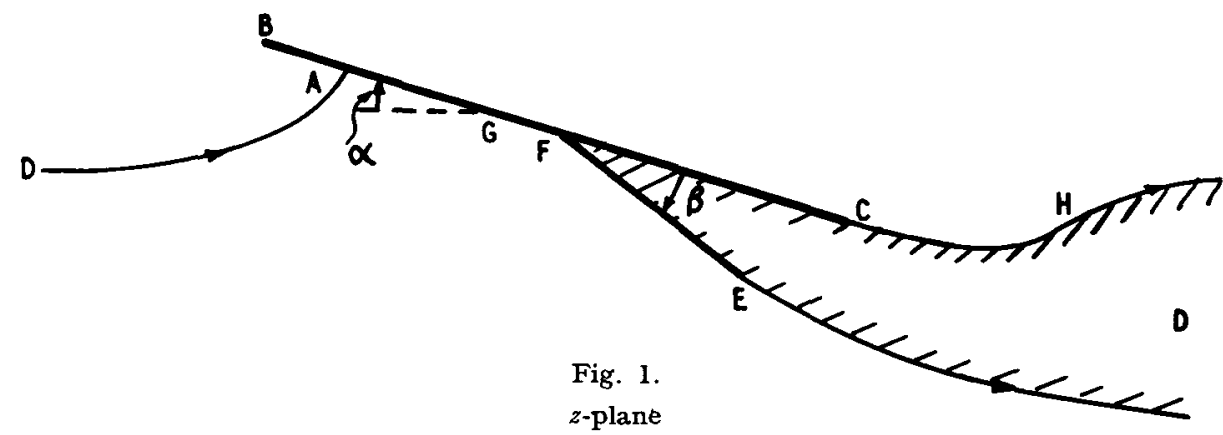

1 A similar model is used by L. C. Woods in ref. 1 which is at present in the press. The mathematical approach used by Woods is different to that followed herein and explicit results are given only when the incidence and flap deflection are small. Results obtained by the present method for these conditions are shown to agree with those of Woods (see $\S 5$ ). The author is grateful to Professor Woods for lending him the manuscript so enabling this comparison to be made. 


$$
Q=\log \left(-U \frac{d z}{d w}\right)=\log \frac{U}{q}+i \theta
$$

Here $q$ is the fluid speed, $U$ the fluid speed at infinity and $\theta$ the direction of flow.

To obtain the correct mapping it is necessary to use experience to specify the appropriate traces in the $Q$-plane of the wing, flap and wake boundary. These are shown in Fig. 2 and involve the following assumptions which will be justified a posteriori,

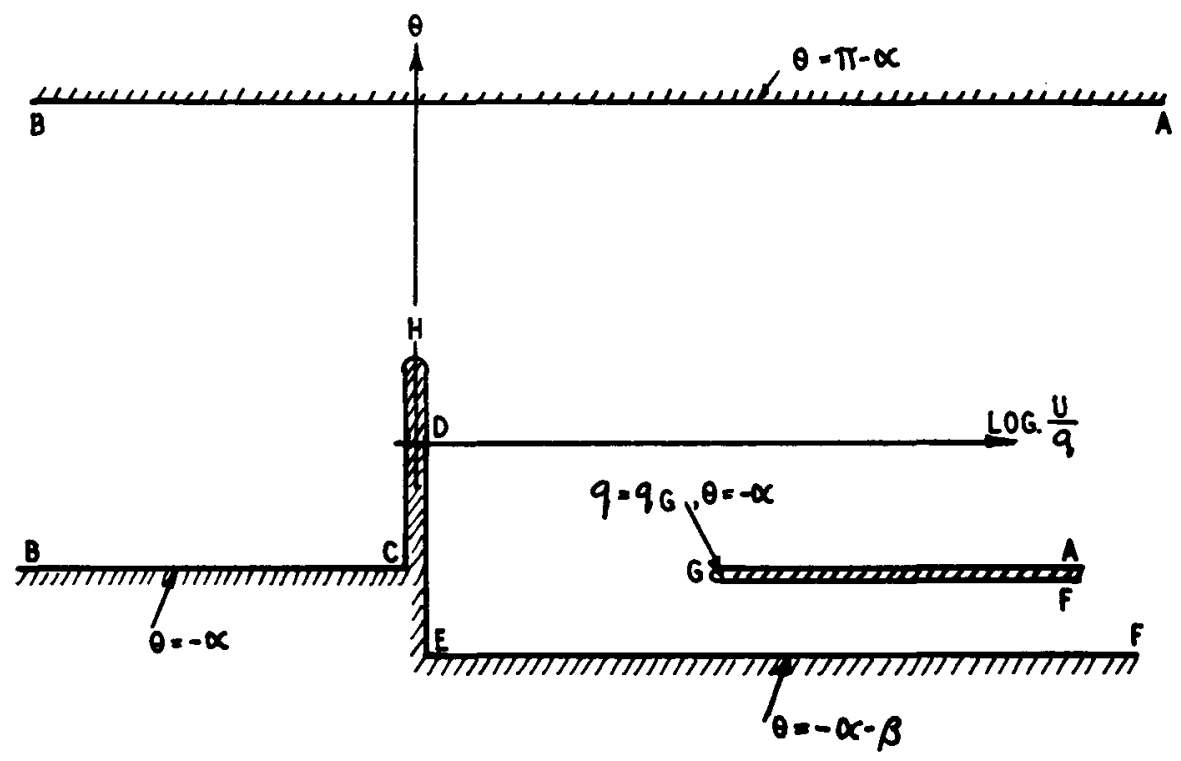

Fig. 2.

$Q$-plane

(i) that $q$ varies monotonically with distance along $A B, B C$ and $F E$,

(ii) that along $A F, q$ increases to a local maximum, $q_{G}$, at $G$ and then decreases to zero at $F$,

(iii) that there is a point of inflection, $H$, on the upper free streamline but no point of inflection on the lower free stream-line.

Assumption (iii) leads to the wake width at infinity being infinite (see $\S 4$ ). In the special case when $H$ coincides with $D$, the point at infinity, the wake width at infinity is zero (see alsc $\S 4$ ). If $H$ is on the lower free stream-line then the two free streamslines intersect leading to a physically impossible wake.

The unhatched portion of the $Q$-plane corresponds to the unhatched portion of the $z$-plane and is mapped onto the upper half of the $\zeta$-plane (Fig. 3) by the Schwarz-Christoffel transformation, 


$$
\begin{aligned}
\frac{d Q}{d \zeta} & =\frac{K(\zeta-h)(\zeta-g)}{(\zeta-b)(\zeta-f)\left(\zeta^{2}-1\right)^{\frac{7}{2}}} \\
& =K\left\{1-\frac{(g-b)(h-b)}{(f-b)(\zeta-b)}-\frac{(g-f)(f-h)}{(f-b)(\zeta-f)}\right\} \frac{1}{\left(\zeta^{2}-1\right)^{\frac{1}{t}}},
\end{aligned}
$$

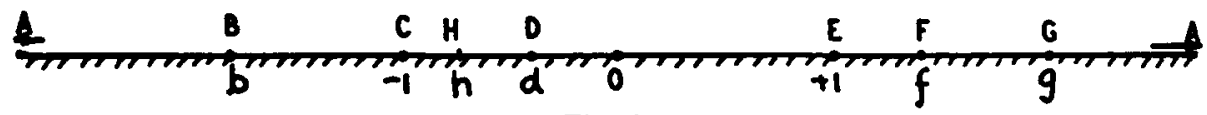

Fig. 3.

$\zeta$-plane

where $K$ is a constant and $b, f, g$ and $h$ are the points on the real $\zeta$-axis to which $B, F, G$ and $H$ map respectively. This equation may be integrated to give

$$
\begin{aligned}
Q= & K \log \left\{\zeta+\left(\zeta^{2}-1\right)^{\mathbf{1}}\right\}+ \\
& +\frac{K(g-b)(h-b)}{(f-b)\left(b^{2}-1\right)^{\mathbf{t}}} \log \left\{\frac{1-b \zeta-\left(b^{2}-1\right)^{\mathbf{t}}\left(\zeta^{2}-1\right)^{\mathbf{t}}}{\zeta-b}\right\} \\
& -\frac{K(g-f)(f-h)}{(f-b)\left(f^{2}-1\right)^{\mathbf{t}}} \log \left\{\frac{1-f \zeta+\left(f^{2}-1\right)^{\mathbf{t}}\left(\zeta^{\mathbf{2}}-1\right)^{\mathbf{t}}}{\zeta-t}\right\}+L,
\end{aligned}
$$

where the principle values of the logarithms have been taken and the branch of $\left(\zeta^{2}-1\right)^{1}$ is that which behaves as $\zeta$ for $\zeta$ large. In this equation and wherever else they occur explicitly, $\left(b^{2}-1\right)^{\mathbf{1}}$ and $\left(t^{2}-1\right)^{\mathbf{t}}$ are taken to denote positive real numbers.

It follows from eqn (3) that all the point to point correspondences shown in Figs. 2 and 3 are satisfied by the transformation

$$
\begin{aligned}
Q= & \log \left\{\zeta+\left(\zeta^{2}-1\right)^{\mathbf{t}}\right\}+\log \left\{\frac{1-b \zeta-\left(b^{2}-1\right)^{\mathbf{t}}\left(\zeta^{2}-1\right)^{\mathbf{t}}}{\zeta-b}\right\} \\
& -\frac{\beta}{\pi} \log \left\{\frac{1-t \zeta+\left(f^{2}-1\right)^{\mathbf{1}}\left(\zeta^{2}-1\right)^{\mathbf{t}}}{\zeta-f}\right\}-i(\alpha+\beta),
\end{aligned}
$$

provided that

(8) $-\alpha-\beta-\gamma=-\operatorname{arcos} h+\operatorname{arcos} \frac{1-b h}{h-b}-\frac{\beta}{\pi} \operatorname{arcos} \frac{1-f h}{h-f}$ 
and

$$
\begin{aligned}
\log \frac{U}{q_{G}}= & \log \left\{g+\left(g^{2}-1\right)^{\mathbf{t}}\right\}+\log \left\{\frac{1-b g-\left(b^{2}-1\right)^{\frac{1}{t}}\left(g^{2}-1\right)^{\mathbf{t}}}{g-b}\right\} \\
& -\frac{\beta}{\pi} \log \left\{\frac{-1+g f-\left(f^{2}-1\right)^{\frac{1}{\mathbf{t}}\left(g^{2}-1\right)^{\mathbf{t}}}}{g-f}\right\} .
\end{aligned}
$$

In these equations $\alpha$ is the wing incidence, $\beta$ the flap deflection, $\gamma$ the value of $\theta$ at the point $H$ (see Fig. 1) and $d$ is the point on the real $\zeta$-axis to which the point $D$ maps.

It follows from eqns (1) and (4) that

$$
\frac{d w}{d z}=\frac{-U e^{i(\alpha+\beta)}\left\{\frac{1-f \zeta+\left(f^{2}-1\right)^{\frac{1}{(}\left(\zeta^{2}-1\right)^{\frac{1}{1}}}}{\zeta / \pi}\right\}^{\beta-f}}{\left\{\zeta+\left(\zeta^{2}-1\right)^{\frac{1}{t}}\right\}\left\{\frac{1-b \zeta-\left(b^{2}-1\right)^{\frac{1}{(}\left(\zeta^{2}-1\right)^{\mathbf{t}}}}{\zeta-b}\right\}}
$$

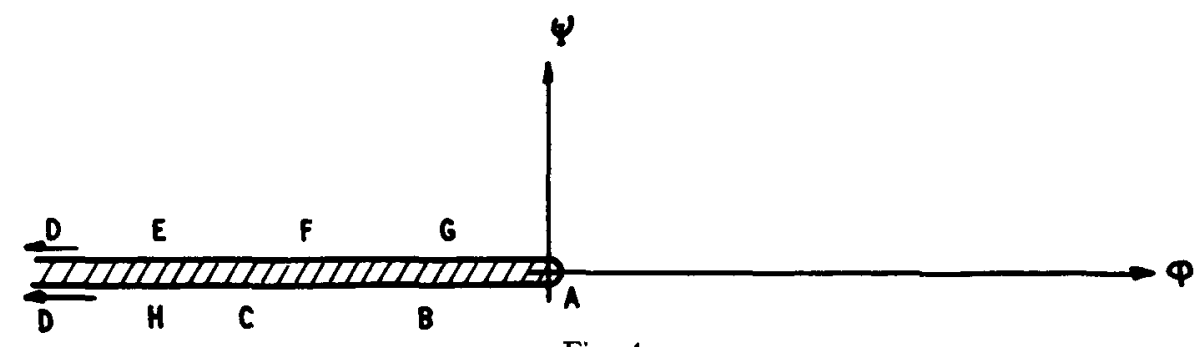

Fig. 4.

w-plane

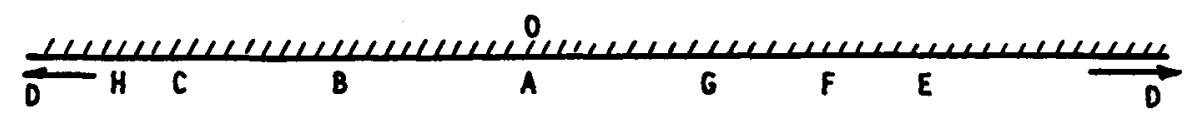

Fig. 5.

$t$-plane

To determine $d w / d \zeta$ consider the trace (Fig. 4) of the wing, flap and wake boundary in the $w=\phi+i \psi$ plane where $\phi$ is the velocity potential and $\psi$ is the stream function. The unhatched portion of this plane is mapped onto the lower half of the $t$-plane (Fig. 5) by the transformation,

$$
w=-U t^{2}
$$

and thence onto the upper half of the $\zeta$-plane (Fig. 3) by

Thus

$$
t=-\frac{1}{\zeta-d} \text {. }
$$

$$
\frac{d w}{d \zeta}=\frac{2 U}{(\zeta-d)^{3}}
$$


The lengths of the various parts of the wing-flap combination and also the shapes of the free stream-line may be obtained by performing numerically the integration

$$
z=\int \frac{d z}{d w} \frac{d w}{d \zeta} d \zeta
$$

where the path of integration is the real $\zeta$-axis. The values of $d z / d w$ and $d w / d \zeta$ there are obtained from eqns (10) and (11) respectively.

For a particular solution, the values of the various parameters must be such that eqns (5) to (9) are satisfied. These equations constitute 5 relationships between the 9 parameters $b, d, f, g, h, \alpha, \beta, \gamma$ and $U / q_{G}$. Thus the family of solutions is a 4 parameter one. This is as expected as 4 parameters are also needed to specify the relative ${ }^{2}$ ) geometry of a certain wing-flap combination: the incidence, $\alpha$, the flap angle, $\beta$, the length of the flap as a fraction of that of the wing, and the position where the flap meets the wing.

A convenient way of satisfying eqns (5) to (9) is as follows:

(i) $\alpha, \beta, b$ and $f$ are selected,

(ii) $g$ is eliminated from eqns (5) and (6) to give

$$
\frac{\beta}{\pi} \frac{\left(f^{2}-1\right)^{t}}{f-h}+1=\frac{\left(b^{2}-1\right)^{t}}{h-b}
$$

which is solved for $h$.

(iii) Eqns (5), (7), (8) and (9) are solved in turn for $g, d, \gamma$ and $U / q_{G}$ respectively.

\section{Calculation of lift and drag}

It follows from Birkhoff and Zarantonello, 1957, that the lift force, $Y$, and the drag force, $X$, acting on the wing flap combination are given by

$$
X-i Y=\frac{i}{2} \rho U^{2} C \text { onj } \int_{\varnothing} \frac{d z}{d w} \frac{d w}{d \zeta} d \zeta-\frac{1}{2} i \rho \int_{\varangle} \frac{d w}{d z} \frac{d w}{d \zeta} d \zeta
$$

where the path of integration, $\mathscr{C}$, is a small semi-circle lying above the point $\zeta=d$ and described in the $+^{\mathrm{ve}}$ sense.

Now from eqn (1)

$$
\frac{d}{d \zeta}\left(\frac{d z}{d w}\right)=\frac{d z}{d w} \frac{d Q}{d \zeta}
$$

and

$$
\frac{d^{2}}{d \zeta^{2}}\left(\frac{d z}{d w}\right)=\frac{d z}{d w}\left\{\frac{d^{2} Q}{d \zeta^{2}}+\left(\frac{d Q}{d \zeta}\right)^{2}\right\}
$$

2 The absolute scale in the physical plane follows automatically from eqns (10) and (11). It could be altered by including a constant real factor in the R.H.S. of eqn (11). 
Thus near $\zeta=d$

$$
\begin{aligned}
\frac{\dot{d} z}{d w}=-\frac{1}{U}\left\{1+Q^{\prime}(d)(\zeta-d)+\frac{1}{2}\left\{Q^{\prime \prime}(d)+\left[Q^{\prime}(d)\right]^{2}\right\}\right. & (\zeta-d)^{2} \\
& \left.+O(\zeta-d)^{3}\right\}
\end{aligned}
$$

where the dashes denote differentiation rert $\zeta$, and from eqn (4)

$$
Q^{\prime}(d)=-\frac{i}{\left(1-d^{2}\right)^{3}}\left\{1-\frac{\left(b^{2}-1\right)^{3}}{d-b}+\frac{\beta}{\pi} \frac{\left(f^{2}-1\right)^{2}}{f-d}\right\}
$$

and

$$
\begin{aligned}
& Q^{\prime \prime}(d)=-\frac{i d}{\left(1-d^{2}\right)^{1}}\left\{1-\frac{\left(b^{2}-1\right)^{\mathbf{t}}}{d-b}+\frac{\beta}{\pi} \frac{\left(f^{2}-1\right)^{\dagger}}{f-d}\right\} \\
& -\frac{i}{\left(1-d^{2}\right)^{3}}\left\{\frac{\left(b^{2}-1\right)^{\ddagger}}{(d-b)^{2}}+\frac{\beta}{\pi} \frac{\left(f^{2}-1\right)^{\dagger}}{(f-d)^{2}}\right\} \text {. }
\end{aligned}
$$

It follows from eqns (11) and (14) to (17) and also the residue theorem that

$$
X=\frac{\pi \rho U^{2}}{\left(1-d^{2}\right)}\left\{1-\frac{\left(b^{2}-1\right)^{1}}{d-b}+\frac{\beta}{\pi} \frac{\left(f^{2}-1\right)^{1}}{f-d}\right\}^{2}
$$

and

$$
\begin{aligned}
Y= & \pi \rho U^{2}\left\{\frac{d}{\left(1-d^{2}\right)^{1}}\left[1-\frac{\left(b^{2}-1\right)^{1}}{d-b}+\frac{\beta}{\pi} \frac{\left(f^{2}-1\right)^{3}}{f-d}\right]\right. \\
& \left.+\frac{1}{\left(1-d^{2}\right)^{\frac{1}{2}}}\left[\frac{\left(b^{2}-1\right)^{1}}{(d-b)^{2}}+\frac{\beta}{\pi} \frac{\left(f^{2}-1\right)^{1}}{(f-d)^{2}}\right]\right\} .
\end{aligned}
$$

It is noted from eqns (13) and (18) that when $h=d$ the drag, $X$, is zero. It will be shown below that in this case the wake width at infinity is zero, whereas generally it is infinite.

\section{Asymptotic geometry of free stream-lines}

It follows from eqns (11) and (15) that near infinity

$$
\text { (20) } z=\frac{1}{(\zeta-d)^{2}}+\frac{2 Q^{\prime}(d)}{\zeta-d}-\left\{Q^{\prime \prime}(d)+\left[Q^{\prime}(d)\right]^{2}\right\} \log (\zeta-d)+O(\zeta-d) \text {. }
$$

Now on the upper free stream-line $\zeta-d$ is real and negative and on the lower free stream-line it is real and positive. Also from eqns (16) and (18)

and from eqns (17) and (19)

$$
Q^{\prime}(d)=-i\left\{\frac{X}{\pi \rho U^{2}}\right\}^{t}
$$

$$
Q^{\prime \prime}(d)=-i\left\{\frac{Y}{\pi \rho U^{2}}\right\} .
$$


Thus it follows from eqn (20), that on the upper free stream-line

$$
y=2\left\{\frac{X}{\pi \rho U^{2}}\right\}^{\ddagger} x^{\mathbf{t}}-\frac{Y}{2 \pi \rho U^{2}} \log x+\frac{X}{\rho U^{2}}+O\left\{\frac{\log x}{x^{\mathbf{t}}}\right\}
$$

and on the lower free stream-line

$$
y=-2\left\{\frac{X}{\pi \rho U^{2}}\right\}^{\ddagger} x^{\ddagger}-\frac{Y}{2 \pi \rho U^{2}} \log x+O\left\{\frac{\log x}{x^{3}}\right\} .
$$

It is seen that in the general case the wake width increases like $x^{\mathbf{t}}$ and the downward displacement of its centre line like $\log x$. In the special case when $h=d$ the drag, $X$, is zero (see eqns. (13) and (18)) and then the wake width tends to zero as $x$ increases.

\section{Approximate solutions when $\alpha$ and $\beta$ are small}

Suppose that:

(i) $\alpha, \beta$ and $d$ are small and of the same order, $O(\alpha)$,

(ii) $b$ is large - ve and $O(1 / \alpha)$,

and (iii) $t$ is $O(1)$.

Then referring to eqn (7),

$$
\begin{gathered}
\operatorname{arcos} d=\frac{\pi}{2}-\operatorname{arsin} d=\frac{\pi}{2}-d+O\left(\alpha^{3}\right), \\
\operatorname{arcos} \frac{1-b d}{d-b}=\frac{\pi}{2}+\frac{1}{b}-d+O\left(\alpha^{3}\right)
\end{gathered}
$$

and

$$
\begin{aligned}
\operatorname{arcos} \frac{1-f d}{d-f} & =\operatorname{arcos}\left(-\frac{1}{f}\right)+O(\alpha) \\
& =\pi-\operatorname{arcos}\left(\frac{1}{f}\right)+O(\alpha) .
\end{aligned}
$$

Thus eqn (7) may be written

$$
-\frac{1}{b}=\alpha+\frac{\beta}{\pi} \operatorname{arcos}\left(\frac{1}{f}\right)+O\left(\alpha^{2}\right) .
$$

It follows from eqns (18), (19) and (21) that

$$
X=\pi \rho U^{2}\left\{\left(\frac{\beta}{\pi}\right)^{2}\left(1-\frac{1}{f^{2}}\right)+O\left(\alpha^{3}\right)\right\}
$$

and

$$
Y=\pi \rho U^{2}\left\{\alpha+\frac{\beta}{\pi} \operatorname{arcos}\left(\frac{1}{f}\right)+\frac{\beta}{\pi f}\left(1-\frac{1}{f^{2}}\right)^{t}+O\left(\alpha^{2}\right)\right\} .
$$


It can be shown from eqn (12), that for the cases being considered, the geometry of the wing-flap combination is as shown in Fig. 6, the errors

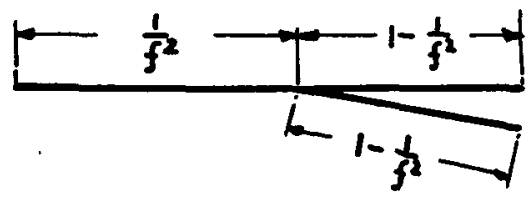

Fig. 6.

being at most $O(\alpha)$. The chord is unity and the length of the flap is equal to the distance of its hinge from the trailing edge of the wing, which is so for cases of practical interest. It follows from eqns (22) and (23) that

$$
C_{D}=\frac{X}{\frac{1}{2} \rho U^{2}}=\frac{2}{\pi} \beta^{2} C_{f}+O\left(\alpha^{3}\right)
$$

and

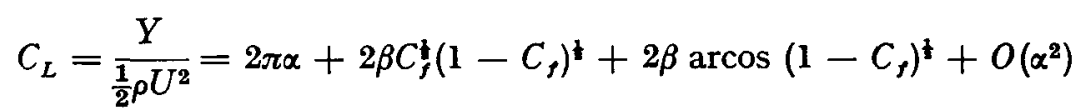

where $C_{f}=1-1 / t^{2}$ is the length of the flap. These values of $C_{D}$ and $C_{L}$ agree with those given by Woods on $\mathbf{P 4 4 5}$ of ref. 2, which is at present in the press. The author is grateful to Professor Woods for lending him the manuscript, thus enabling the comparison to be made.

Now from eqn (25)

$$
\left(\frac{\partial C_{L}}{\partial \beta}\right)_{\alpha=0, \beta=0}=2 C_{f}^{t}\left(1-C_{f}\right)^{t}+2 \operatorname{arcos}\left(1-C_{f}\right)^{t}+O(\alpha) .
$$

The values of this quantity for a wing fitted with a plain flap have been given by Glauert in ref. 3, and are precisely twice those given by eqn (26) above. Thus, according to the present theory, for equal values of $\beta$ the split flap should be exactly half as efficient in producing lift increments as is the plain flap.

\section{Numerical calculations}

The solutions for which $h=d$ have been shown to have zero wake width at infinity (and zero drag) and thus may be considered as constituting a "boundary" to solutions having physical significance. It is therefore of some interest to investigate the wing-flap geometries that give rise to such solutions. The procedure described in $\S 2$ was used to determine this geometry for two cases when $\alpha=10^{\circ}$ and $\beta=60^{\circ}$, and the main results are given in Table II and Fig. 7. 
TABLE 2

Principal results of calculations for two cases when $\alpha=10^{\circ}, \beta=60^{\circ}$ and $X=0(h=d)$

\begin{tabular}{|l|l|l|l|l|l|}
\hline$A B$ & $A F$ & $F E$ & $B C$ & $C_{L}$ & $q_{G} / U$ \\
\hline 0.126 & 0.972 & 0.203 & 3.033 & 1.10 & 0.475 \\
1.022 & 0 & 1.478 & 8.834 & 0.99 & 0 \\
\hline
\end{tabular}
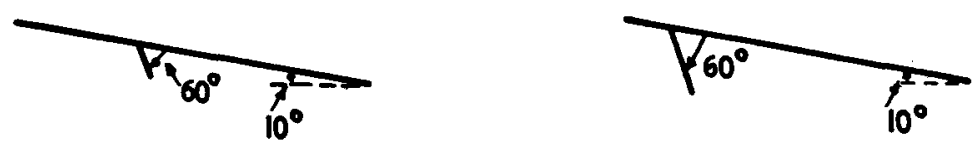

Fig. 7.

Fig. 7 shows that in both cases the trailing edge of the flap does not protrude as far below the trailing edge of the wing as is so for configurations of practical interest. It would thus appear that configurations of practical interest give rise to solutions having non-zero drag and infinite wake width at infinity (see $\S 4$ ).

\section{Comparison with experiment}

The values of $\left(\partial C_{L} / \partial \beta\right)_{\alpha=0, \beta=0}$ given by eqn (26) are shown in Fig. 8. Reference 4 gives experimental values of $C_{D}$ and $C_{L}$ when $\alpha$ and $\beta$ are small for three aerofoils having thickness/chord ratios, $t / c$, of $0.30,0.21$ and 0.12 . Values of $\left(\partial C_{L} / \partial \beta\right)_{\alpha=0, \beta=0}$ were derived from these results and are included in Fig. 8.

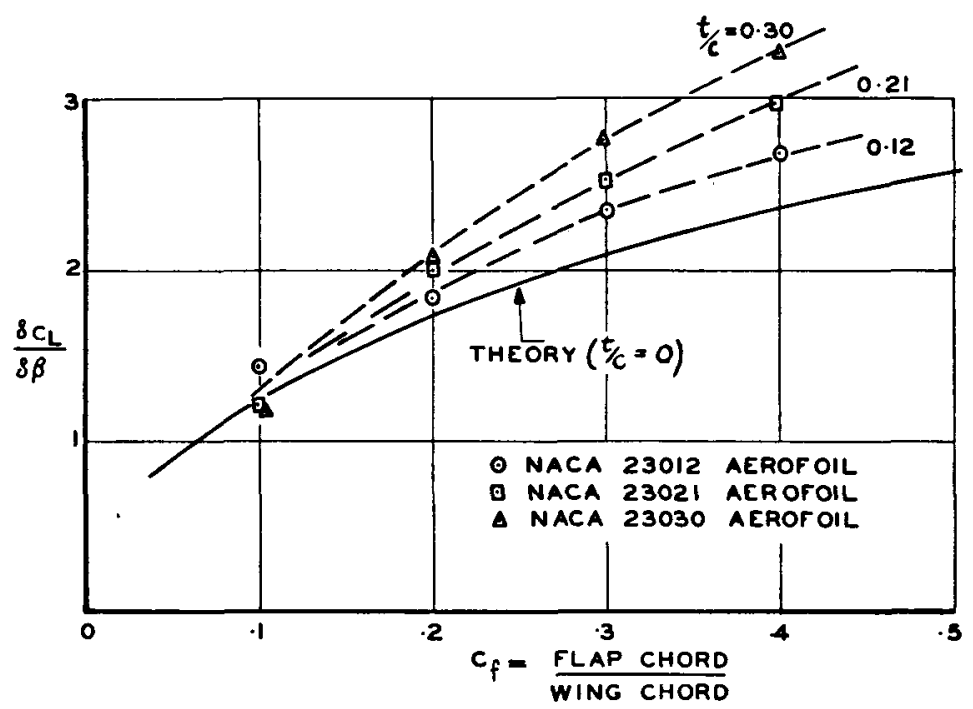

Fig. 8. 
The figure shows that the experimental values exceed the theoretical ones by amounts that decrease with $t / c$ and suggests that satisfactory agreement between theory and experiment would be obtained for very thin aerofoils. The theoretical values of $C_{D} / \beta^{2}$ (see eqn (24)) were found to be up to $70 \%$ less than the experimental values, so that the theory is not satisfactory for predicting values of $C_{D}$.

\section{References}

[1] Woods, L. C., The theory of subsonic plane flow C.U.P. (in press).

[2] Birkhoff, G. and Zarantonello, E. H., Wakes Jets and Cavities, Academic Press 1957. Chap. IV.

[3] Glauert, H., Theoretical Relationships for an aerofoil with hinged flap. Aero Res. Cttee. R \& M. 1095. 1927.

[4] Wenzinger, C. J. and Harris, T. A., Wind Tunnel Investigation of N.A.C.A. 23012, 23021 and 23030 airfoils with various sizes of split flaps. N.A.C.A. Report 668, 1939.

Aeronautical Research Laboratories,

Department of Supply, Melbourne. 